\title{
Low Temperature Growth of Hydrogenated Silicon Prepared by PECVD from Argon Diluted Silane Plasma
}

\author{
Rachid Amrani $^{12^{*}}$, Pascale Abboud $^{1}$, Larbi Chahed ${ }^{2}$, Yvan Cuminal ${ }^{1}$ \\ ${ }^{1}$ IES, UMR, Université Montpellier II, Place Eugène Bataillon, Montpellier, France \\ ${ }^{2}$ LPCMME, Département de Physique, Université d'Oran ES-Sénia, Oran, Algérie \\ Email: *rachidamrani2002@yahoo.fr, rachid.amrani@ies.univ-montp2.fr
}

Received October 7, 2012; revised November 14, 2012; accepted November 23, 2012

\begin{abstract}
In order to contribute to the understanding of the optoelectronics properties of hydrogenated nanocrystalline silicon thin films, a detailed study has been conducted. The samples were deposited by $13.56 \mathrm{MHz}$ PECVD (Plasma-Enhanced Chemical Vapor Deposition) of silane argon mixture. The argon dilution of silane for all samples studied was $96 \%$ by volume. The substrate temperature was fixed at $200^{\circ} \mathrm{C}$. The influence of depositions parameters on optical proprieties of samples was studied by UV-Vis-NIR spectroscopy. The structural evolution was studied by Raman spectroscopy and $\mathrm{X}$-ray diffraction (XRD). Intrinsic-layer samples depositions were made in this experiment in order to obtain the transition from the amorphous to crystalline phase materials. The deposition pressure varied from $400 \mathrm{mTorr}$ to $1400 \mathrm{mTorr}$ and the rf power from 50 to $250 \mathrm{~W}$. The structural evolution studies show that beyond $200 \mathrm{~W}$, we observed an amorphous-nanocrystalline transition, with an increase in crystalline fraction by increasing $\mathrm{rf}$ power and working pressure. Films near the amorphous to nanocrystalline transition region are grown at reasonably high deposition rates $(\sim 10 \AA / \mathrm{s})$, which are highly desirable for the fabrication of cost effective devices. The deposition rate increases with increasing rf power and process pressure. Different crystalline fractions $(21 \%$ to $95 \%)$ and crystallite size $(6-16 \mathrm{~nm})$ can be achieved by controlling the process pressure and rf power. These structural changes are well correlated to the variation of optical proprieties of the thin films.
\end{abstract}

Keywords: Silicon; PECVD; Deposition Rate; Amorphous Nanocrystalline Transition; Argon; Low Temperature

\section{Introduction}

For high quality solar cell applications, materials with high optical absorption, high carrier mobility and low fabrication cost are demanded. Crystalline silicon (C-Si), the most popular electronic material, has an indirect bandgap and, hence, poor optical absorption. On the other hand, hydrogenated amorphous silicon (a-Si:H) has high optical absorption, but it suffers from low carrier mobility, photo-induced degradation also named StablerWronski effect [1,2] and, hence, poor optoelectronic properties. Recently, thin film hydrogenated nanocrystalline silicon (nc-Si:H) deposited by Plasma Enhanced Chemical Vapor Deposition (PECVD) emerged as a material for large-area electronics applications [3-6]. The fabrication cost for nc-Si:H optoelectronic applications is expected to be low, since thin films of nc-Si:H can be deposited directly over large-area substrates using the same fabrication facilities well established for a-Si:H devices. Plasma deposited hydrogenated nanocrystalline silicon (nc-Si:H) offers the possibilities of high carrier

${ }^{*}$ Corresponding author. mobility and stability against Staebler-Wronski effects [6-9].

An enhanced optical absorption has been observed in nanocrystalline silicon films $[10,11]$. The nanocrystalline can absorb the photons of weak energies whereas amorphous silicon effectively absorbs the photons of high energies. The optical absorption of nc-Si:H is evidently highly dependent on the crystalline fraction. In contrast to the a-Si:H film, the nc-Si:H film shows an increased absorption below $1.8 \mathrm{eV}$ and a reduced absorption above $2.0 \mathrm{eV}$. With decreasing crystallinity, the absorption coefficient decreases at lower photon energies nearly up to $1.4 \mathrm{eV}$, and increases beyond $2.0 \mathrm{eV}$ [12].

The absorption coefficient of nc-Si:H is almost an order of magnitude higher than that of c-Si. Thus, only $\sim 2$ $\mu \mathrm{m}$ thick layer is necessary for the nanocrystalline cell compared to the $>100 \mu \mathrm{m}$ thick wafer used for a c-Si cell.

The plasma-enhanced chemical vapor deposition (PECVD) method from silane plasma is widely used to deposit the amorphous and nanocrystalline hydrogenated silicon Si:H $[13,14]$. The importance of the effects of the gas dilution on the kinetics of powder formation is im- 
portant when one wants to transfer one process from one dilution to another. This is particularly important for example when we want to increase the deposition rate. When silane $\left(\mathrm{SiH}_{4}\right)$ is diluted with hydrogen, the device quality nc-Si:H films prepared by PECVD method at optimized deposition parameters show lower deposition rate. In the present paper, the $\mathrm{Si}: \mathrm{H}$ samples were prepared from $\left(\mathrm{SiH}_{4}+\mathrm{Ar}\right)$ plasma in a conventional capacitive coupled rf (13.56 MHZ) PECVD system. The structural evolution of the samples was investigated by means of X-ray diffraction and Raman scattering measurements. The optical characterization of these thin films was also appraised by UV-Vis-NIR spectroscopy in order to study the influence of deposition parameters on the optical properties of thin films. The purpose of this work is to investigate the optical and structural properties of the thin $\mathrm{Si}: \mathrm{H}$ films to be able to apply them for the photovoltaic applications with high deposition rate.

\section{Experimental Procedure}

The argon diluted hydrogenated silicon $\mathrm{Si}: \mathrm{H}$ thin films were deposited in a conventional $\mathrm{rf}(13.56 \mathrm{MHz})$ PECVD chamber at substrate temperature of $200^{\circ} \mathrm{C}$. The deposition pressure varied from $400 \mathrm{mT}$ orr to $1000 \mathrm{mTorr}$, at various rf power $(50,100,200$ and $250 \mathrm{~W})$. Samples were grown on glass substrates. The magnitude of the residual stress depends on the thin film and the substrate properties. The residual stress was qualitatively estimated. A big residual stress results usually in fracturing and emitting layer from the substrate. In order to avoid this complexity, a $\mathrm{SiO}_{2}$ underlayer was deposited. As consequence, no layer delamination was observed.

The structural properties of the samples were investigated by means of X-ray diffraction in standard $(\theta-2 \theta$ scans) configuration. The XRD studies are performed at grazing angle of incidence using $\mathrm{CuK} \alpha \mathrm{X}$-ray radiation $(\lambda=$ $1.54056 \AA$ ). The crystallinity was also characterized by Raman scattering measurements. All Raman spectra were measured with an Ar-Ion laser at a wavelength of 473.5 $\mathrm{nm}$. The power of the Raman laser was kept about $2 \mathrm{~mW}$ to avoid laser induced crystallization on the films. The Raman spectra of nc-Si:H consisted of a narrow line at $520 \mathrm{~cm}^{-1}$ due to a crystalline phase and a broad line around $480 \mathrm{~cm}^{-1}$ due to an amorphous phase. The third component between 500 and $510 \mathrm{~cm}^{-1}$ is due to the band dilation at grain boundaries. The optical constants of these films are estimated with the help of UV-Vis-NIR transmission measurements in the range of $300-2700$ $\mathrm{nm}$. The samples images were taken by Scanning electron Microscope (SEM).

\section{Results and discussion}

The variation of deposition rate plotted as a function of argon dilution in silane as shown in Figure 1, with 1000 mTorr working pressure, $100 \mathrm{~W}$ rf power, $50 \mathrm{sccm}$ silane flow and with different argon flow of 6, 50 and 1200 sccm. It is well known that the gas phase particles appearance time in silane argon plasma decreases when the gas ration argon to silane increases [15-19]. This is particularly important to increase the deposition rate. It is seen from Figure 1, for 1 minute deposition time, the deposition rate increases from $\sim 2 \AA / \mathrm{s}$ to $\sim 16 \AA / \mathrm{s}$, when the argon dilution in silane increases from $10 \%$ to $96 \%$. But with increase in the deposition time to 10 minutes, the growth rate decreases to $8 \AA / \mathrm{s}$. Indeed, due to the presence of powder trapped in the plasma, film growth is prevented.

In order to obtain high deposition rate and without dust trapped in the plasma, the silane flow was decreased to $10 \mathrm{sccm}$ and the argon flow was fixed at $250 \mathrm{sccm}$. The deposition time was fixed at 15 minutes.

As shown in Figure 2(a), the deposition rate increases from $\sim 6 \AA / \mathrm{s}$ to $\sim 7.5 \AA / \mathrm{s}$ when the process pressure increases from 400 mTorr to 1000 mTorr. With further increase in process pressure to 1400 mTorr, the deposition rate decreases.

The impingement rate of gas molecules is given by;

$$
\frac{P}{\sqrt{2 \pi m k_{B} T}}
$$

with $P$ is the process pressure, $\mathrm{m}$ is the molecular mass, $k_{B}$ is Boltzmann's constant and $T$ is the gas temperature [20]. Thus, with increase in process pressure the impingement rate of silane increases. As a result, the number of film-forming radicals and hence the deposition rate increases.

However, the powders electrostatically trapped in the plasma prevent the films growth. So, for deposition without dust trapped in the reactor, the working pressure must be below the critical value of 1000 mTorr. As

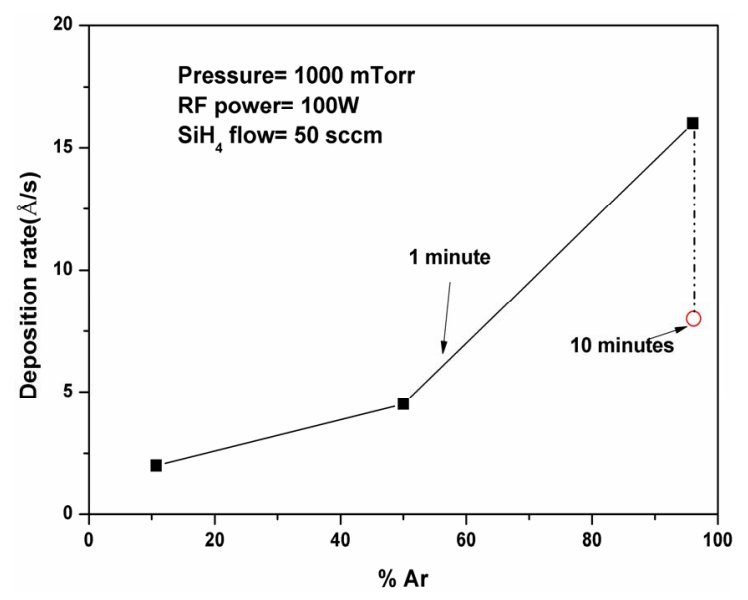

Figure 1. Variation of deposition rate plotted as a function of argon dilution in silane. 


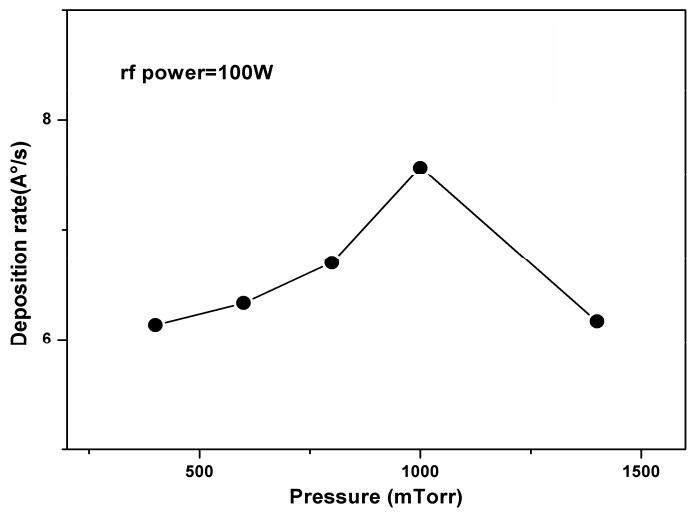

(a)

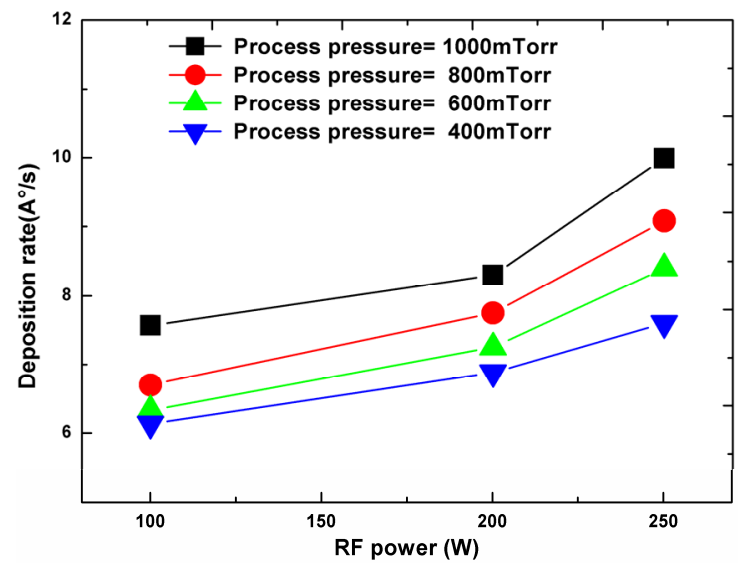

(b)

Figure 2. Variation of deposition rate plotted as a function of process pressure (a) and rf power (b).

shown in Figure 2(b), at certain working pressure, it is observed that with increase in rf power, the deposition rate increases. To achieve a better understanding of the optical proprieties of $\mathrm{Si}: \mathrm{H}$, the optical transmission of films was measured by UV-Vis-NIR spectrophotometer. The films thickness $t$ and the refraction index $n$ were determined using the method proposed by Swanepoel [21].

Detailed analysis of the refractive index spectra was performed using the model suggested by Wemple and Didomenico $[11,22,23]$. At energies below than of the optical bandgap, the refractive index is related to the square of the photon energy $(\hbar \omega)^{2}$ by:

$$
n^{2}(\hbar \omega)=1+\frac{E_{M} E_{D}}{E_{M}^{2}-(\hbar \omega)^{2}}
$$

The plot of $1 /\left[n^{2}(\hbar \omega)-1\right]$ versus $(\hbar \omega)^{2}$ allows the determination of the average gap $E_{M}$, the energy of dispersion $E_{D}$, and static refractive index $n_{0}$. The results of this analysis are reported in Table $\mathbf{1}$.

The dispersion energy $E_{D}$, characteristic of the material, represents the oscillator force of the inter-band optical transition and depends on the average number of co- ordination. The greater value of $E_{D}$ obtained for the samples, indicates a greater coordinance average number, which is associated with a reduction of porosity in these films and consequently a reduction in the disordered fields in the vicinity of structural heterogeneities (microcavities). This result is in accord with the values of the refraction index. The static index $n_{0}$, index of refraction corresponding to zero energy, represents the compactness of material. The static refractive index increases with increase in process pressure indicating increase in the material density in the film.

Micro-Raman spectroscopy has been widely used as a powerful technique to characterize deposited thin layers. Figure 3 shows Raman spectra of $\mathrm{Si}: \mathrm{H}$ films deposited at various process pressure and rf power. For samples deposited below $200 \mathrm{~W}$, a broad peak located around 480 $\mathrm{cm}^{-1}$, characteristic of a completely amorphous structure. Beyond $200 \mathrm{~W}$ rf power and beyond 800 mTorr pressure deposition, the (TO) band can be correctly fitted using three Gaussian components centered around 480, 510 and $520 \mathrm{~cm}^{-1}$, suggesting the presence in these films a mixture of amorphous as well as crystalline structure with different grain size [23-26]. The mean grain size may be calculated from the formula;

$$
d_{\text {Raman }}=2 \pi(\beta / \Delta \omega)^{1 / 2}
$$

where $\beta=2 \mathrm{~nm}^{2} / \mathrm{cm}$ and $\Delta \omega$ is the peak shift for nanocrystalline as compared to that of c-Si [27].

The crystalline fraction Fc can be estimated from the deconvoluted peaks of Raman spectra, as shown in Figure 4. The first scattering $I_{a}$ in the region of $460-490$ $\mathrm{cm}^{-1}$ comes from the TO vibration modes of amorphous silicon, the intermediate component $I_{b}$ arises near 500 $510 \mathrm{~cm}^{-1}$ due to the band dilation at grain boundaries and the third component $I_{c}$ at $514-520 \mathrm{~cm}^{-1}$ is attributed to the crystalline phase. Considering the intermediate

Table 1. Values of the films thickness $t$, the static refractive index $n_{0}$, the dispersion energy $E_{D}$ and the average gap $E_{M}$, obtained for the films grown at 100 and $200 \mathrm{~W}$ and with

\begin{tabular}{|c|c|c|c|c|c|}
\hline RF power (W) & Pressure (mTorr) & $t(\mathbf{n m})$ & $n_{0}$ & $E_{D}(\mathrm{eV})$ & $E_{M}(\mathrm{eV})$ \\
\hline 100 & 400 & 551.7 & 3.6 & 17.68 & 1.8 \\
\hline 100 & 600 & 569.7 & 3.64 & 18.08 & 1.78 \\
\hline 100 & 800 & 603 & 3.71 & 19.92 & 1.76 \\
\hline 100 & 1000 & 680.4 & 3.73 & 20.26 & 1.8 \\
\hline 200 & 400 & 612 & 3.5 & 17.44 & 1.78 \\
\hline 200 & 600 & 648 & 3.53 & 17.47 & 1.63 \\
\hline 200 & 800 & 697.5 & 3.56 & 17.52 & 1.56 \\
\hline 200 & 1000 & 747 & 3.58 & 17.65 & 1.45 \\
\hline
\end{tabular}
different process pressures. 



Figure 3. Typical Raman spectra obtained in the TO-like mode, for films deposited (a): with deposition pressure of $1000 \mathrm{~m}$ Torr and varying $\mathrm{rf}$ power $(50,100$ and $200 \mathrm{~W})$ and (b): rf power $200 \mathrm{~W}$ and varying pressure $(600,800$ and 1000 mTorr).

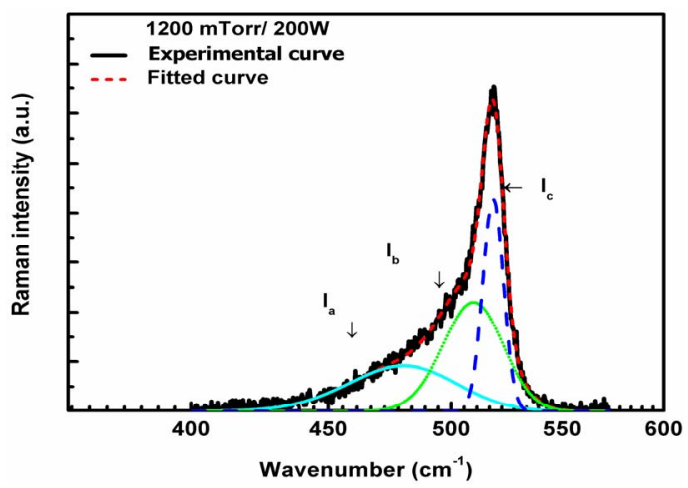

Figure 4. The deconvoluted peaks of Raman spectra for the film deposited at $1200 \mathrm{mT}$ Torr and $200 \mathrm{~W}$.

component as a portion of the crystal, the ratio of the volume fraction of crystalline is defined by $F_{c}=\left(I_{c}+\right.$ $\left.I_{b}\right) /\left(I_{c}+I_{b}+\mu I_{a}\right)$, where $\mu$ is a scattering factor.

As the grain size is about a few nanometers, we can take $\mu \approx 1[28,29]$.

The results for the crystalline fraction and Raman Crystallite size $d_{\text {Raman }}$ are summarized in Table 2.

Additional information about the structural changes of the films is gained from the XRD results. XRD patterns of two typical samples at the same pressure (1400 mTorr) with different rf power, as indicated, are shown in Figure 5. For the sample deposited at $100 \mathrm{~W}$, no crystal grains are detected, indicating that there is no apparent structural evolution in the thin films. When rf power reaches $200 \mathrm{~W}$, diffraction peaks arise. With the increase of $\mathrm{rf}$ power, there appear three peaks symbolizing three different silicon crystalline orientations. The peaks observed at angles of $28^{\circ}, 47^{\circ}$ and $56^{\circ}$ are assigned to $\mathrm{Si}(111)$, $\mathrm{Si}(220)$ and $\mathrm{Si}(311)$ reflection planes of faced-centred cubic silicon, respectively, demonstrating a proper growth of nc-Si:H. Also, the growth of grains in the thin films is multi-oriented. The mean grain size $d_{X R D}$ estimated using the classical Scherrer's formula [30] is also indicated in Table 2.

The average crystallite size increases with increasing working pressure, these results are consistent with Raman scattering results and give further strong support to the formation of nc-Si:H films by PECVD with argon to silane mixture. Crystallite sizes measured by XRD method turned out difference with those measured by Raman method. The difference can be due to the different detection sensitivity of characterization techniques.

Table 2. Raman crystallites size $d_{\text {Raman }}$ XRD average grain size $d_{X R D}$ and crystalline fraction obtained for samples deposited at $200 \mathrm{~W}$ rf power and various working pressure.

\begin{tabular}{ccccc}
\hline RF power (W) Pressure (mTorr) & $\mathrm{d}_{\text {Raman }}(\mathrm{nm})$ & $\mathrm{d}_{\text {XRD }}(\mathrm{nm})$ & $\mathrm{Fc}(\%)$ \\
\hline 200 & 600 & -- & -- & -- \\
200 & 800 & 6.4 & 7.1 & 21 \\
200 & 1000 & 9.8 & 9.5 & 42 \\
200 & 1200 & 11.5 & 12.2 & 61 \\
200 & 1400 & 16.2 & 15.1 & 95 \\
\hline
\end{tabular}

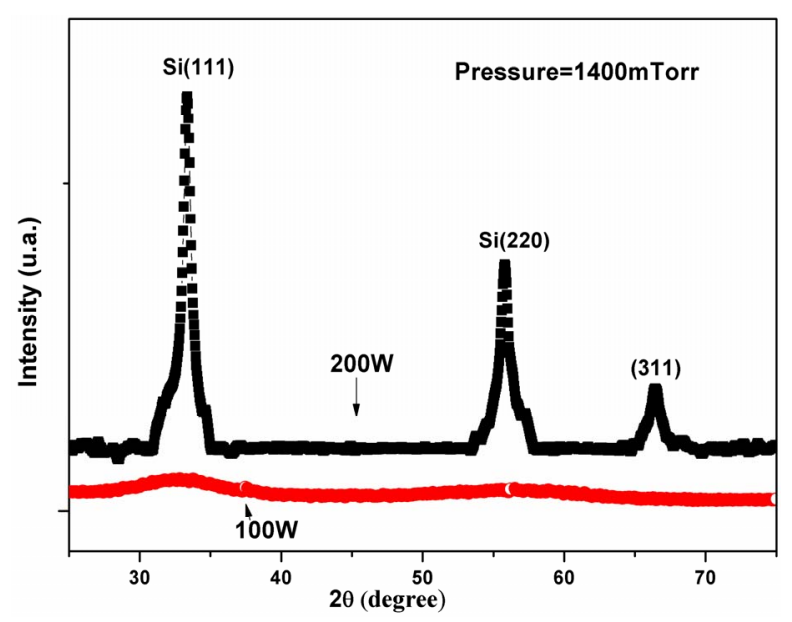

Figure 5. Low angle X-ray diffraction pattern of two typical samples deposited at the same pressure (1400 mTorr) with different $r f$ power $(100$ and $200 \mathrm{~W})$. The spectra are shifted vertically for better clarity. 
SEM images revel significant difference between amorphous and nanocrystalline samples. As shown in Figure 6(a), SEM studies of samples deposited at 400 mTorr and $200 \mathrm{~W}$ show smooth conchoidal surface morphology.

From the cross-sectional SEM on the fractured surfaces of the films also no columnar structure is observed. Figure 6(b) shows SEM image of sample deposited at 1000 mTorr and $200 \mathrm{~W}$. The film has uniformly distributed grains.

The application of Tauc approximation [31] to calculate the optical gap for nanocrystalline silicon is still a subject of debate. Thus, there are several ambiguities about the band gap of nc-Si:H films because the material contains both amorphous and crystalline phases. In the nanocrystalline areas, the indirect band-gap should be around $1.1 \mathrm{eV}$ (close to the crystalline silicon value), while in the amorphous areas, it is around $2 \mathrm{eV}$ (similar to the a-Si:H value, depending on the process parameters).

The average gap $E_{M}$ seems quite suitable to describe the variation of the optical properties of the amorphous and nanocrystalline silicon thin films [11,22,23]. As show in Table 1, for the samples deposited at $100 \mathrm{~W}$, the average gap $\mathrm{E}_{\mathrm{M}}$, is around $1.8 \mathrm{eV}$. But for films grown at $200 \mathrm{~W}$ and beyond process pressure of $800 \mathrm{mTorr}, \mathrm{E}_{\mathrm{M}}$ decreases considerably. This confirms once again, that beyond these deposition parameters, an amorphous to nanocrystalline transition is observed.

As seen from Table 1, the average gap of nc-Si:H films (deposited at $200 \mathrm{~W}$ ) decreases to 1.45 as deposition pressure increases to 1000 mTorr. We believe that the low average gap of nc-Si:H films may be due to the increase in crystalline volume fraction in the film, as revealed by Raman spectroscopic analysis. This inference is further strengthened by the observed variation in static refractive index with process pressure.

\section{Conclusion}

We have shown that hydrogenated nanocrystalline silicon
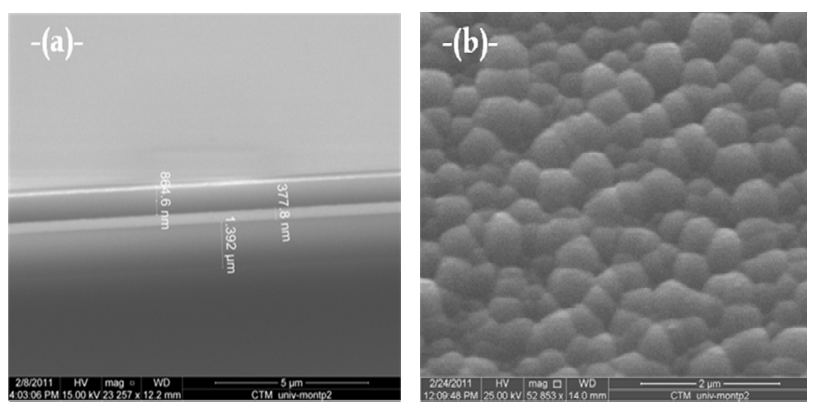

Figure 6. Scanning electron Microscope (SEM) images of (a): Amorphous sample with $\mathrm{SiO}_{2}$ under-layer; and (b): nanocrystalline sample. con (nc-Si:H) films can be prepared with highly argon silane dilution PECVD at high deposition rates and at low substrate temperature $\left(200^{\circ} \mathrm{C}\right)$. Samples obtained have a great compactness. Optical and structural thin films properties of $\mathrm{Si}: \mathrm{H}$ can be tuned by adjusting the deposition conditions. Films with different crystalline fractions and crystallite size are achieved by controlling the process pressure. The ease of depositing films with tunable average band gap and at high deposition rate is useful for photovoltaic applications. Low-temperature processes particularly adequate for large-area devices open up not only very important cost-reduction potential, but also new possibilities such as making semi-transparent or flexible modules.

\section{Acknowledgements}

This work was supported by the grant Averroes Program funded by the European commission.

\section{REFERENCES}

[1] W. E. Spear and P. G. LeComber, "Substitutional Doping of Amorphous Silicon," Solid State Communications, Vol. 17, No. 9, 1975, pp. 1193-1196. doi:10.1016/0038-1098(75)90284-7

[2] D. L. Staebler and C. R. Wronki, "Reversible Conductivity Changes in Discharge-Produced Amorphous Si," Applied Physics Letters, Vol. 31, No. 4, 1977, 3 p. doi:10.1063/1.89674

[3] M. Ito, C. Koch, V. Svrcek, M. B. Schubert and J. H. Werner, "Silicon Thin Film Solar Cells Deposited under $80^{\circ}$ C," Thin Solid Films, Vol. 383, No. 1-2, 2001, pp. 129-131. doi:10.1016/S0040-6090(00)01590-X

[4] A. V. Shah, J. Meier, E. Vallat-Sauvan, N. Wyrsch, U. Kroll, C. Droz and U. Graf, "Material and Solar Cell Research in Microcrystalline Silicon," Solar Energy Materials \& Solar Cells, Vol. 78, No. 1-4, 2003, pp. 469-491.

[5] J. Meier, R. Fluckiger, H. Keppner and A. V. Shah, "Complete Microcrystalline $p-i-n$ Solar Cell-Crystalline or Amorphous Cell Behavior?" Applied Physics Letters, Vol. 65, No. 7, 1994, p. 860. doi:10.1063/1.112183

[6] C. H. Lee, A. Sazonov and A. Nathan, "High-Mobility Nanocrystalline Silicon Thin-Film Transistors Fabricated by Plasma-Enhanced Chemical Vapor Deposition," Applied Physics Letters, Vol. 86, No. 22, 2005, Article ID: 222106. doi:10.1063/1.1942641

[7] I. C. Cheng, S. Allen and S. Wagner, "Evolution of Nanocrystalline Silicon Thin Film Transistor Channel Layers," Journal of Non-Crystalline Solids, Vol. 720, No. 1, 2004, pp. 338-340.

[8] P. R. i Cabarrocas, R. Brenot, P. Bulkin, R. Vanderhaghen, B. Drevillon and I. French, "Stable Microcrystalline Silicon Thin-Film Transistors Produced by the Layer-byLayer Technique," Journal of Applied Physics, Vol. 86, No. 12, 1999, p. 7079. doi:10.1063/1.371795 
[9] A. Shah, E. Vallat-Sauvain, P. Torres, J. Meier, U. Kroll, C. Hof, C. Droz, M. Goerlitzer, N. Wyrsch and M. Vanecek, "Intrinsic Microcrystalline Silicon ( $\mu \mathrm{c}-\mathrm{Si}: \mathrm{H})$ Deposited by VHF-GD (Very High Frequency-Glow Discharge): A New Material for Photovoltaics and Optoelectronics," Materials Science and Engineering: B, Vol. 69-70, 2000, pp. 219-226. doi:10.1016/S0921-5107(99)00299-8

[10] Z. Remes, "Study of Defects and Microstructure of Amorphous and Microcrystalline Silicon Thin Films and Polycrystalline Diamond Using Optical Methods," Ph.D. Thesis, Charles University, Prague, 1999.

[11] R. Amrani, D. Benlekehal, R. Baghdad, D. Senouci, A. Zeinert, K. Zellama, L. Chahed, J. D. Sib and Y. Bouizem, "Low-Temperature Growth of Nanocrystalline Silicon Films Prepared by RF Magnetron Sputtering: Structural and Optical Studies," Journal of Non-Crystalline Solids, Vol. 354, No. 19-25, 2008, pp. 2291-2295. doi:10.1016/j.jnoncrysol.2007.10.044

[12] F. Siebke, S. Yata, Y. Hishikawa and M. Tanaka, "Correlation between Structure and Optoelectronic Properties of Undoped Microcrystalline Silicon," Journal of NonCrystalline Solids, Vol. 227-230, No. 2, 1998, pp. 977981. doi:10.1016/S0022-3093(98)00261-0

[13] A. Matsuda, "Thin-Film Silicon-Growth Process and Solar Cell Application," Japanese Journal of Applied Physics, Vol. 43, 2004, pp. 7909-7920. doi:10.1143/JJAP.43.7909

[14] R. A. Street, "Large Area Electronics, Applications and Requirements," Physica Status Solidi A, Vol. 166, No. 2, 1998, pp. 695-705. doi:10.1002/(SICI)1521-396X(199804)166:2<695::AIDPSSA695>3.0.CO;2-U

[15] J. L. Dorier, C. Hollenstein and A. A. Howling, "Powder Dynamics in Very High Frequency Silane Plasmas," Journal of Vacuum Science \& Technology A, Vol. A10, No. 4, 1992, pp. 1048-1052. doi:10.1116/1.578200

[16] J. L. Dorier, C. Hollenstein and A. A. Howling, "Spatiotemporal Powder Formation and Trapping in Radio Frequency Silane Plasmas Using Two-Dimensional Polarization-Sensitive Laser Scattering," Journal of Vacuum Science \& Technology A, Vol. 13, No. 3, 1995, pp. 918-928. doi:10.1116/1.579852

[17] A. A. Howling, J. L. Dorier, C. Hollenstein, U. Kroll and F. Finger, "Frequency Effects in Silane Plasmas for Plasma Enhanced Chemical Vapor Deposition," Journal of Vacuum Science \& Technology A, Vol. 10, No. 4, 1992, pp. 1080-1085. doi:10.1116/1.578205

[18] J. L. Dorier, "Genèse, Croissance et Conséquences de Particules Dans les Plasmas en Silane à Basse Pression et Basse Température," Ph.D. Thesis, 1996.

[19] A. Bouchoule, "Dusty Plasma: Physics, Chemistry and Technological Impacts in Plasma Processing," Wiley, New York, 1999.
[20] S. Kasap and P. Capper, "Springer Handbook of Electronic and Photonic Materials," Springer Publication, 2006.

[21] R. Swanpoel, "Determination of the Thickness and Optical Constants of Amorphous Silicon," Journal of Physics E: Scientific Instruments, Vol. 16, No. 12, 1983, pp. 1214-1222. doi:10.1088/0022-3735/16/12/023

[22] S. H. Wemple and M. Didomenico, "Behavior of the Electronic Dielectric Constant in Covalent and Ionic Materials," Physical Review B, Vol. 3, No. 4, 1971, pp. 13381351. doi:10.1103/PhysRevB.3.1338

[23] R. Amrani, F. Pichot, J. Podlecki, A. Foucaran, L. Chahed and Y. Cuminal, "Optical and Structural Proprieties of nc-Si:H Prepared by Argon Diluted Silane PECVD," Journal of Non-Crystalline Solids, Vol. 358, No. 17, 2012, pp. 1978-1982. doi:10.1016/j.jnoncrysol.2012.01.022

[24] S. Veprek, F. A Sarott and Z. Iqbal, "Effect of Grain Boundaries on the Raman Spectra, Optical Absorption, and Elastic Light Scattering in Nanometer-Sized Crystalline Silicon," Physical Review B, Vol. 36, No. 6, 1987, p. 3444.

[25] H. S. Mavi, A. K. Shukla, S. C. Abbi and K. P. Jain, "Raman Study of Amorphous to Microcrystalline Phase Transition in cw Laser Annealed a-Si:H Films," Journal of Physic Science, Vol. 66, No. 11, 1989, p. 5322. doi: $10.1063 / 1.343723$

[26] Y. He, Y. Wei, G. Zheng, M. Yu and M. Liu, "An Exploratory Study of the Conduction Mechanism of Hydrogenated Nanocrystalline Silicon Films," Journal of Applied Physics, Vol. 82, No. 7, 1997, p. 3408. doi: $10.1063 / 1.365656$

[27] Y. He, C. Yin, G. Cheng, L. Wang, X. Liu and G. H. Hu, "The Structure and Properties of Nanosize Crystalline Silicon Films," Journal of Applied Physics, Vol. 75, No. 2, 1994, pp. 797-803. doi:10.1063/1.356432

[28] G. Yue, J. D. Lorentzien, J. Lin, D. Han and Q. Wang, "Photoluminescence and Raman Studies in Thin-Film Materials: Transition from Amorphous to Microcrystalline Silicon," Applied Physics Letters, Vol. 75, No. 4, 1988, pp. 492-494. doi:10.1063/1.124426

[29] D. Beeman, R. Tsu and M. F. Tporpe, "Structural Information from the Raman Spectrum of Amorphous Silicon," Physical Review B, Vol. 32, No. 2, 1985, pp. 874878. doi:10.1103/PhysRevB.32.874

[30] P. Scherrer, "Bestimmung der Grösse und Derinneren Struktur von Kolloidteilchen Mittels Röntgenstrahlen," Nachrichten von der Gesellschaft der Wissenschaften zu Göttingen, Mathematisch-Physikalische Klasse, Vol. 26, No. 1, 1918, pp. 98-100.

[31] J. Tauc, "Optical Properties of Solids," Abeles, North Holland, Amsterdam, 1972. 